'Научно-исследовательский институт акушерства, гинекологии и репродуктологии им. Д.О. Отта, Санкт-Петербург, Россия

${ }^{2}$ Национальный медицинский исследовательский центр им. В.А. Алмазова, Санкт-Петербург, Россия ${ }^{3}$ Северо-Западный государственный медицинский университет им. И.И. Мечникова, Санкт-Петербург, Россия

В настоящее время в России и в мире отсутствует единый подход к диагностике и лечению субклинического гипотиреоза (СГТ) при планировании и во время беременности. Обусловлено это отличиями в референсных значениях тиреотропного гормона (ТТГ) для различных популяций и сроков беременности, влиянием маркеров аутоиммунного процесса в щитовидной железе (ЩЖ) на риски осложнений беременности, укоренившимся в России представлением о фиксированных нормах ТТГ во время беременности, предложенных Американской тиреоидологической ассоциацией (АТА) в 2011 и Европейской тиреоидологической ассоциацией (ЕТА) в 2014 гг., а также с отсутствием до недавнего времени Российских клинических рекомендаций по данному вопросу. Свой вклад вносит и мультидисциплинарный подход к таким больным, включающий наблюдение врачами нескольких специальностей - гинекологом, эндокринологом и, во многих случаях, репродуктологом, у каждого из которых может оказаться свое представление о целевых показателях тиреоидных гормонов для данной пациентки. Как следствие, имеет место проблема отсутствия единого подхода к терапии СГТ во время беременности в различных медицинских учреждениях, избыточной лекарственной, финансовой и психологической нагрузки на беременных.

Согласно последним рекомендациям АТА 2017 г. и проекту российских клинических рекомендаций по лечению гипотиреоза 2019 г., решение вопроса о терапии левотироксином при СГТ во время беременности во многих случаях остается на усмотрение лечащего врача. В данном обзоре систематизируется накопленный к настоящему времени научный и клинический опыт, который позволит специалистам, встречающимся с проблемами СГТ во время беременности, принять решение о диагностике и терапии данного заболевания, основываясь на принципах доказательной медицины.

КЛЮЧЕВЫЕ СЛОВА: субклинический гипотиреоз; тиреотропный гормон; беременность; тироксин.

\title{
DIAGNOSTIC AND TREATMENT OF SUBCLINICAL HYPOTHYROIDISM IN PREGNANT AND PLANNING PREGNANCY PATIENTS: MODERN VIEW OF THE PROBLEM
}

\author{
(c) Ekaterina S. Shilova ${ }^{1,2 *}$, Natalya V. Borovik', Polina V. Popova², Maria I. Yarmolinskaya ${ }^{1,3}$
}

${ }^{1}$ Research Institute of Obstetrics, Gynecology, and Reproductology named after D.O. Ott, Saint Petersburg, Russia

${ }^{2}$ Almazov National Medical Research Center, Saint Petersburg, Russia

${ }^{3}$ North-Western State Medical University named after I.I. Mechnikov, Saint Petersburg, Russia

There is no universal diagnostic and treatment strategy of subclinical hypothyroidism (SHT) in pregnant women and those who are planning pregnancy due to differences in population-specific and trimester-specific thyroid-stimulating hormone (TSH) reference values, influence of thyroid autoimmunity markers on pregnancy outcomes, adherence to fixed universal cutoff concentrations TSH in Russia and multidisciplinary team care with different treatment opinions involving. An absence of universal approach to SHT leads to excess treatment, financial and psychological burden on pregnant women.

Up to American Thyroid Association (ATA) Guidelines 2017 and project of Clinical Guidelines of Russian Association of Endocrinologists 2019 a treatment strategy of SHT should be left up to the endocrinologist to choose. In our paper we systematize global scientific and clinical experience of planning and management pregnancies with SHT to help physicians to choose treatment option based on principals of evidence-based medicine.

KEYWORDS: subclinical hypothyroidism; thyroid-stimulating hormone; thyroxin; pregnancy. 
Субклинический гипотиреоз (СГТ) во время беременности определяется как превышение допустимых при беременности значений тиреотропного гормона (ТТГ) при нормальном уровне гормонов щитовидной железы (ЩЖ) $[1,2]$. СГТ ассоциирован с рисками для здоровья матери и ребенка, преимущественно с преждевременными родами и самопроизвольными абортами [2-4]. Маркеры аутоиммунного процесса в ЩЖ присутствуют у 2-17\% беременных женщин $[2,4]$ и, как изолированно, так и совместно с изменением тиреоидного статуса, ассоциированы с осложнениями наступления и течения беременности, такими как самопроизвольные аборты [5-8], невынашивание [9] и преждевременные роды $[8,10-12]$.

Встречаемость и показания к терапии тиреоидной патологии во время беременности зависят от исследуемой популяции, срока беременности, наличия маркеров аутоиммунного процесса в ЩЖ, а также от принятых референсных значений для ТТГ и свободного тироксина (св.Т4), в связи с чем фиксированные референсные значения для уровня ТТГ и св.Т4 во время беременности В настоящее время подвергнуты сомнению $[2,13]$. В настоящее время в России и в мире отсутствует единый подход к диагностике и лечению СГТ, в связи с чем большое значение имеет информированность врачей, встречающихся с проблемами СГТ у беременных, о современных представлениях о подходах к данной проблеме. Определение корректных показаний для терапии СГТ во время беременности необходимо, с одной стороны, для предотвращения осложнений беременности, с другой для предотвращения необоснованной лекарственной, психологической и финансовой нагрузки на беременных женщин и систему здравоохранения.

\section{ФИЗИОЛОГИЧЕСКИЕ ИЗМЕНЕНИЯ ТИРЕОИДНОГО СТАТУСА ВО ВРЕМЯ БЕРЕМЕННОСТИ}

Беременность предполагает физиологические изменения уровня ТТГ и гормонов ЩЖ. Во-первых, схожая структура ТТГ и хорионического гонадотропина человека (ХГЧ) способствует стимуляции рецепторов ТТГ в ЩЖ и увеличению синтеза тиреоидных гормонов, а также, по принципу обратной связи, снижению уровня ТТГ в І триместре беременности. Во-вторых, под воздействием эстрогенов в печени усиливается синтез тироксинсвязывающего глобулина, обладающего высокой аффинностью к тироксину. Как следствие, с 7-й недели беременности нарастает уровень общего Т4 в крови беременной. В-третьих, работа дейодиназы 3-го типа в плаценте повышает распад тироксина и трийодтиронина, что также требует увеличения синтеза тиреоидных гормонов во время беременности [14-16]. Наконец, увеличение фильтрационной функции почек приводит к повышению экскреции йода с мочой во время беременности и требует большего потребления йода беременной, в том числе для обеспечения возросшей потребности в тиреоидных гормонах [14-16]. Кроме того, определение св.Т4 автоматическими иммунохимическими анализаторами затруднено во время беременности в связи с повышением уровня тироксинсвязывающего глобулина и снижением концентрации альбумина в крови [2].
Основной причиной СГТ в развитых странах считается аутоиммунный тиреоидит [16], и у всех женщин с уровнем ТТГ выше 2,5 мЕД/л необходимо определять уровень антител к тиреопероксидазе (АТкТПО) $[2,13]$. Однако стоит принять во внимание супрессию иммунной системы во время беременности, в связи с чем титр тиреоидных антител снижается в среднем на $60 \%$ во второй половине беременности [17]. В исследовании Korevaar T.M. et al. было показано, что женщины с уровнем АТкТПО, превышающим 92-й процентиль, имели больший уровень ТТГ и больший риск увеличения ТТГ >2,5 мЕд/л. Было также отмечено, что женщины с повышенным уровнем АТкТПО хуже отвечают на стимуляцию ХГЧ во время беременности. Чем выше уровень АТкТПО, тем хуже ответ в виде изменения уровня сВ.Т4 и ТТГ на стимуляцию ХГЧ [12].

На способность ЩЖ адаптироваться к возросшим потребностям в ее работе во время беременности влияют уровень потребления йода, индекс массы тела (ИМТ), этническая принадлежность, уровень ХГЧ и другие плацентарные факторы.

Йод - основной компонент тиреоидных гормонов, необходимый для их синтеза. Выраженный дефицит потребления йода во время беременности может привести к гипотиреозу и кретинизму ребенка [18]. В развивающихся странах основной причиной СГТ во время беременности считается недостаточное потребление йода [16]. В России ряд регионов признаны йододефицитными. По данным Д.Е. Соболевой и соавт., при обследовании 103 беременных женщин, проживающих в Санкт-Петербурге, медиана йодурии составила 117,00 мкг/л (75,00-170,90), что соответствует недостатку йода. Процент новорожденных, имеющих уровень ТТГ более 5 мЕД/л, за 2013-2014 гг. составил 6,9\%, что также соответствует легкому дефициту йода в регионе [19]. Всем беременным женщинам, проживающим в регионах, подозрительных на йододефицит, рекомендован прием препаратов йода при планировании, в течение беременности и грудного вскармливания $[1,2]$. Этническая принадлежность также может влиять на функцию щЖ во время беременности за счет генетических, культурных, пищевых различий $[20,21]$. Отчасти с этим связаны различия в референсных интервалах для тиреоидных гормонов во время беременности в разных странах. Больший ИМТ ассоциирован с большим уровнем ТТГ и свободного трийодтиронина (св.Т3), меньшим уровнем св.Т4 [22, 23]. В исследовании Han C. et al. верхняя граница нормы ТTГ для женщин с ИМТ 20-25 кг/м² составила 2,86 мЕД/л, а для женщин с ИМТ более 30 кг/м² - 3,5 мЕД/л [22].

Быстрое нарастание уровня ХГЧ в І триместре беременности приводит к повышению уровня св.Т4 и, по принципу обратной связи, к снижению уровня ТТГ $[14,16]$. Однако, по данным Korevaar T.I. et al., тиреоидный ответ на стимуляцию ХГЧ значительно ухудшается у женщин с маркерами аутоиммунного процесса в ЩЖ, такими как повышение уровня АТкТПО. Исследователями была получена отрицательная корреляция между уровнем ТТГ и ХГЧ и положительная - между уровнем св.Т4 и ХГЧ у женщин с АТкТПО <60 мЕД/л, в отличие от тех, у кого уровень АТкТПО был более 60 мЕД/л [24]. В исследовании Hou Y. et al. также был получен неадек- 
ватный тиреоидный ответ на стимуляцию ХГЧ во время беременности у женщин с повышенным уровнем АТкТПО ( $\geq 53$ мЕД/л) [25]. Кроме ХГЧ, В плаценте синтезируются факторы ангиогенеза, такие как растворимая fms-подобная тирозинкиназа-1 (sFlt-1) и плацентарный фактор роста (ПФР). По данным Korevaar T.l. et al., повышение концентрации sFlt-1 ассоциировано с 2,4-кратным повышением риска СГТ и 3-кратным повышением риска гипотироксинемии, а высокий уровень ПФР - с 1,8-кратным риском гипотироксинемии во время беременности [26].

\section{РЕФЕРЕНСНЫЕ ЗНАЧЕНИЯ ТТГ}

Ключевым вопросом в диагностике СГТ при планировании и во время беременности является использование корректных референсных значений. Верхняя граница нормы ТТГ вне беременности зависит от лаборатории и, как правило, составляет 3,5-4,0 мЕД/л. При впервые выявленном повышении уровня ТТГ и нормальном св.Т4 рекомендуется провести повторное определение ТТГ, св.T4 и АТкТПО через 2-3 мес для подтверждения наличия у пациента стойкого СГТ [13].

Физиологические изменения во время беременности, как уже обсуждалось выше, приводят к снижению уровня ТТГ и предполагают определение нормы ТТГ во время беременности [2]. В 2011 г. АТА предложила использовать фиксированные триместр-специфические нормы ТТГ во время беременности: 0,1-2,5 мЕд/л в I триместре, 0,2-3,0 мЕд/л во ІІ триместре и 0,3-3,0 мЕд/л в III триместре в случае отсутствия возможности определения специфичных для региона или медицинского центра норм [27]. Похожие фиксированные референсные значения ТТГ были рекомендованы ЕTA: 0,1-2,5 мЕд/л в I триместре, 0,2-3,0 мЕд/л во II триместре и 0,3-3,5 мЕд/л в III триместре [1]. Однако, по данным исследований, встречаемость тиреоидной патологии во время беременности зависит от исследуемой популяции, срока беременности, наличия маркеров аутоиммунного процесса в ЩЖ. При использовании фиксированных норм ТТГ во время беременности выявляемость СГТ резко возросла и достигла 6,1-27,8\% В некоторых популяциях [28-30]. В случае применения специфичных для популяции и триместра беременности норм ТТГ распространенность СГТ составляет только 2-4\%. При анализе результатов 14 крупных популяционных исследований было показано, что в 12 из них (63 362 участников) за верхнюю границу нормы ТТГ были приняты значения 2,5 или 3,0 мЕД/л, тогда как верхние границы специфических референсных значений для этих популяций составили 2,63-4,68 мЕД/л [31]. Например, специфические референсные значения ТТГ для Италии составили 0,09 $(0,06-0,12)-3,16(3,05-3,29)$ в I триместре; 0,25 (0,11-0,30)-3,55 (3,34-3,73) во II триместре и 0,42 (0,15-0,48)-3,93 (3,80-4,08) в III триместре беременности. При использовании фиксированных норм ТТГ СГТ был бы диагностирован у 8,8, 6,1 и 6,6\% женщин В I, II и III триместрах соответственно в сравнении с 2,5\% во всех трех триместрах при использовании локального референса [28]. По нашим данным, специфические референсные значения ТТГ в І триместре беременности для женщин, проживающих в Санкт-Петербурге, составили 0,07-4,40 мЕд/л. Распространенность СГТ среди
503 включенных в исследование беременных составила 16,9\%, исходя из диагностического критерия ТТГ >2,5 мЕд/л, и 3,8\% - при использовании рассчитанного в ходе исследования референсного интервала [32].

Все это позволяет говорить о значительной гипердиагностике СГТ при использовании унифицированных норм ТТГ и, как следствие, необоснованном лечении женщин по всему миру. В связи с этим в 2017 г. АТА подвергла сомнению фиксированные референсные значения для уровней ТТГ и св.Т4 во время беременности [2]. АТА продолжает рекомендовать рассчитывать специфические для популяции и триместра беременности нормы ТТГ, а при отсутствии таковых предлагает использовать значение 4,0 мЕд/л в качестве верхней границы ТТГ [2].

В России до недавнего времени отсутствовали национальные рекомендации по диагностике и лечению гипотиреоза во время беременности. По нашим представлениям, наиболее распространены фиксированные нормы ТTГ, соответствующие рекомендациям АTA 2011 и ЕTA 2014 гг. В настоящее время ведется подготовка клинических рекомендаций по гипотиреозу, проект которых опубликован на официальном сайте Эндокринологического научного центра [13]. Подход к диагностике СГТ, согласно данному проекту, соответствует представлениям АТА 2017 г. и основан не только на уровне ТТГ, но и наличии АТкТПО [13].

\section{ДИАГНОСТИКА И ЛЕЧЕНИЕ СУБКЛИНИЧЕСКОГО ГИПОТИРЕОЗА НА ПРЕГРАВИДАРНОМ ЭТАПЕ}

Манифестный гипотиреоз негативно влияет на репродуктивную функцию женщины. Например, при уровне ТТГ >15 мЕд/л нарушения менструального цикла имели место у $68 \%$ в сравнении с $12 \%$ у эутиреоидных женщин [33]. Влияние СГТ на репродуктивные возможности женщины не столь очевидно. По некоторым данным, повышение ТТГ наблюдается чаще среди женщин, испытывающих трудности с зачатием [34, 35]. По данным Abalovich M. et al., СГТ встречался чаще среди женщин, страдающих от бесплодия, в сравнении с группой контроля (13,9 и 3,9\% соответственно) [35]. По результатам ретроспективного исследования Feldthusen A.D. et al., повышение уровней ТТГ и АТкТПО ассоциировано со снижением фертильности [36]. По данным Рорре К. et al., напротив, повышение ТТГ >4,2 мЕд/л среди женщин с бесплодием встречается не чаще, чем в группе контроля [37]. В проспективном когортном исследовании Powden T.C. et al., включившем более 1000 женщин, уровень ТТГ > 2,5 мЕд/л не был ассоциирован со скоростью наступления беременности и риском самопроизвольных абортов, в том числе и у женщин с повышенным уровнем АТкТПО [38]. Таким образом, по мнению большинства специалистов, отсутствуют достаточные данные в пользу негативного влияния СГТ на возможность наступления беременности $[2,39]$.

Показания к терапии левотироксином при планировании беременности и ее эффективность в настоящий момент также являются дискутабельными. По мнению ATA, на момент 2017 г. не получено достаточно данных, чтобы рекомендовать терапию левотироксином женщинам с нормальным уровнем ТТГ и повышенным уровнем 
АТкТПО при планировании беременности. Однако в 2019 г. опубликовано двойное слепое плацебо-контролируемое исследование Dhillon-Smith R.K. et al., включившее 952 планирующих беременность женщин с ТТГ в диапазоне 0,44-3,63 мЕд/л и повышенным уровнем АТкТПО, проходящих лечение от бесплодия или имевших один самопроизвольный выкидыш и более в анамнезе. Участницы исследования были разделены на две группы: 476 женщин получали плацебо, другие 476 - 50 мкг левотироксина. Значимых различий в частоте наступления беременности в течение 12 мес, исходах беременностей, в том числе риске преждевременных родов, самопроизвольных абортов, неонатальных исходов между группами получено не было [40]. Результаты данного обширного исследования могут склонить мировое сообщество рекомендовать воздержаться от терапии левотироксином в подобном случае.

В настоящий момент АТА рассматривает возможность инициирования терапии левотироксином при СГТ без АТкТПО у женщин, планирующих естественное наступление беременности [2]. Уместно будет напомнить, что СГТ вне беременности соответствует повторному повышению ТТГ выше лабораторного референса, а не уровня ТТГ > 2,5 мЕД/л, как можно нередко встретить в клинической практике. В проекте Российских клинических рекомендаций по лечению гипотиреоза этот вопрос, к сожалению, не рассматривается.

У пациентов, нуждающихся во вспомогательных репродуктивных технологиях (ВРТ), лечение левотироксином при СГТ увеличивает шансы на наступление беременности и рождение живых детей $[2,41]$. По данным рандомизированного исследования Kim C.Y. et al., включившего 64 участницы с бесплодием и уровнем ТТГ >4,5 мЕд/л, частота имплантаций эмбрионов была выше $(26,9 \%$ и 14,9\%, p=0,044), самопроизвольных абортов ниже (0\% и 33,3\%, $\mathrm{p}=0,021)$, число рожденных живых детей больше $(53,1 \%$ и 25,0\%, $p=0,039)$ в группе женщин, получавших лечение левотироксином, по сравнению с группой контроля. Группы были сопоставимы по возрасту, ИМТ, длительности бесплодия, уровню АТкТПО [41]. Соответственно, для женщин, планирующих использование ВРТ, АТА рекомендует терапию СГТ независимо от уровня АТкТПО с достижением целевого ТТГ <2,5 мЕд/л с умеренным уровнем доказательности, а также допускает терапию левотироксином эутиреоидных женщин с повышенным уровнем АТкТПО с низким уровнем доказательности [2]. Однако по данным относительно крупного (600 участников) исследования Wang H. et al., лечение левотироксином женщин с уровнем ТТГ 0,5-4,78 мЕд/л и повышенным уровнем АТкТПО перед вступлением в протокол ЭКО не дает преимуществ по сравнению с группой контроля [42]. С учетом этих данных не исключен пересмотр подхода к терапии тиреоидными гормонами эутиреоидных женщин с маркерами аутоиммунного процесса [43].

\section{РИСКИ ОСЛОЖНЕНИЙ БЕРЕМЕННОСТИ ПРИ СУБКЛИНИЧЕСКОМ ГИПОТИРЕОЗЕ}

Если манифестный гипотиреоз очевидно ассоциирован с риском преждевременных родов и когнитивными нарушениями у детей $[2,44-46]$, вследствие чего требует незамедлительного начала терапии левотироксином, консенсус в отношении рисков СГТ во время беременности в настоящее время не достигнут $[1,2,13]$.

По мнению ряда исследователей, СГТ может быть ассоциирован с риском самопроизвольных абортов $[2,6,47]$. В проспективном когортном исследовании Liu H. et al. принимали участие 3315 женщин, включенных в исследование на сроке 4-8 нед беременности. Риск самопроизвольного аборта был значительно выше у женщин с уровнем ТТГ 5,22-10,0 мЕд/л при наличии АТкТПО или их отсутствии по сравнению с женщинами с ТТГ $<2,5$ мЕд/л без АТкТПО (7,1\% и 2,2\%; отношение шансов (ОШ) 3,40; доверительный интервал (ДИ) 1,62-7,15; $\mathrm{p}=0,002$ и 15,2\% и 2,2\%; ОШ 9,56; ДИ 3,76-24,28; $p=0,000$ соответственно) [6]. В когортном исследовании Schneuer F.J. et al. (2801 женщина в I триместре беременности) повышение ТТГ выше 95-го процентиля ассоциировано с самопроизвольными абортами (ОШ 3,66; 95\% ДИ 1,59-8,44) [48]. Необходимо отметить, что, по данным ряда исследований, риск самопроизвольного аборта возрастает уже при уровне ТТГ > 2,5 мЕд/л [3, 47-50]. В метаанализе Maraka S. et al., включающем 18 когортных исследований, СГТ был определен как уровень ТТГ, превышающий референсные значения при нормальном уровне тироксина, или повышение уровня ТТГ в рамках 2,5-5 мЕд/л. Женщины с СГТ имели повышенный риск самопроизвольного аборта (ОР 2,01; 95\% ДИ 1,66-2,44), отслойки плаценты (ОР 2,14; 95\% ДИ 1,23-3,70), преждевременного излития околоплодных вод (ОР 1,43; ДИ 1,04-1,95), перинатальной смертности (ОР 2,58; ДИ 1,41-4,73) [3]. В исследование Wang S. et al. были включены 756 беременных на сроке до 12 нед. При СГТ (ТТГ 22,5 мЕд/л) самопроизвольные аборты встречались значительно чаще, чем при эутиреозе $(15,48 \%$ vs $8,86 \% ; p=0,03)$ [49]. Однако в этих исследованиях не проводилась оценка уровня АТкТПО, тогда как наличие антитиреоидных антител у беременной повышает риски прерывания беременности на ранних сроках [5-8, 50]. B метаанализ Chen L. et al. включены 22 исследования женщин с эутиреозом и умеренным СГТ (ТТГ 0,3-5,0 мЕд/л). Ассоциация между аутоиммунными процессами в ЩЖ и риском самопроизвольного аборта была выявлена в 8 исследованиях случай-контроль (ОШ 2,55; 95\% ДИ 1,42-4,57; P=0,002) и в 14 когортных исследованиях (ОШ 2,31; 95\% ДИ 1,90-2,82; $\mathrm{P}<0,000$ ) [5]. Это подтверждает и исследование Seungdamrong A. et al., в котором принимали участие 1468 женщин с бесплодием. Частота зачатий, беременностей, самопроизвольных абортов и рождения живых детей не различалась между женщинами с уровнем ТТГ $\geq 2,5$ и ТТГ $<2,5$ мЕД/л. Женщины с повышенным уровнем АТкТПО имели сопоставимую частоту зачатия (33,3\% vs. 36,3\%), но выше частоту самопроизвольных абортов (43,9\% vs. $25,3 \% ; p=0,02)$ и ниже частоту рождения живых детей (17,1\% vs. $25,4 \%$; $\mathrm{p}=0,14)$, чем женщины без повышенного уровня АТкТПО. Применение моделей логистической регрессии подтвердило повышение риска самопроизвольных абортов у женщин с повышенным уровнем АТкТПО (ОШ 2,17; 95\% ДИ 1,12-4,22) [7]. По данным Lopez-Tinoco C. et al., из 435 женщин с СГТ (ТТГ 3,86-10 мЕД/л) в І триместре беременности у 17\% был повышенный уровень АТкТПО. Повышение уровня АТкТПО ассоциировано с 10,25-кратным риском самопроизвольных абортов [50]. 
Также есть данные относительно рисков преждевременных родов при СГТ в начале беременности $[4,8$, $12,48,51]$. По данным Schneuer F.J. et al., повышение уровня ТТГ выше 95-го процентиля в I триместре беременности ассоциировано с преждевременными родами до 37 нед (ОШ 2,59; 95\% ДИ 1,21-5,53) [48]. В ретроспективном исследовании Arbib N. et al., включившим 4504 женщин, было показано, что риск преждевременных родов до 34-й недели беременности выше у женщин с уровнем ТТГ 2,5-4,0 мЕд/л и с ТТГ >4,0 мЕд/л по сравнению с беременными с уровнем ТТГ $<2,5$ мЕд/л В I триместре беременности (ОШ 1,81; 95\% ДИ 1,0-3,28 и ОШ 2,33; 95\% ДИ 1,11-4,42 соответственно) [51]. Однако в данных исследованиях опять же не проводилась оценка уровня АТкТПО, наличие которых повышает риски преждевременных родов [4, 10, 11]. По результатам метаанализа, включившего 47045 беременных, риск преждевременных родов был выше у женщин с СГТ (ТТГ выше 97,5 процентиля), чем при эутиреозе (6,1\% и 5,0\% соответственно; разница рисков 1,4\% [95\% ДИ 0-3,2\%]; ОШ 1,29 [95\% ДИ 1,01-1,64]), а повышение ТТГ на каждое стандартное отклонение (CO) было ассоциировано с большим риском преждевременных родов (разница рисков 0,2\% [95\% ДИ 0-0,4\%] на 1-СО; ОШ 1,04 [95\% ДИ 1,00-1,09] на 1-СО). При этом риск преждевременных родов оказался выше у женщин с повышенным уровнем АТкТПО по сравнению с теми, у кого уровень АТкТПО был в пределах референсных значений (6,6\% и 4,9\% соответственно; разница рисков 1,6\% [95\% ДИ 0,7-2,8\%]; ОШ 1,33 [95\% ДИ 1,15-1,56]) [4]. В другом метаанализе, включившем 7 исследований и 23000 пациентов с различным тиреоидным статусом, также была выявлена ассоциация между наличием маркеров аутоиммунного процесса в ЩЖ и преждевременными родами (ОШ =1,67; 95\% ДИ 1,44-1,94; $p<0,001$ ) [10].

По данным крупного метаанализа Derakshan et al. (36 исследований, 48145 участниц), СГТ ассоциирован с риском рождения маловесного плода $(11,8 \%$ и 10,0\%; 95\% ДИ 0,43-4,81; ОШ 1,24, 1,04-1,48; $p=0,015$ ) [52]. В более ранних и меньших по объему исследованиях такой взаимосвязи получено не было [3, 48, 49, 53].

Данные относительно ассоциации повышения уровня ТТГ и других осложнений беременности: гестационной артериальной гипертензии [3, 49, 53, 54], преэклампсии [3, 54], гестационного сахарного диабета (ГСД) $[3,55]$, преждевременного излития околоплодных вод $[3,53]$, синдрома задержки роста плода $[3,53]$ противоречивы и требуют проведения дальнейших исследований. В настоящий момент не складывается представление о взаимосвязи СГТ с данными осложнениями беременности.

Что касается нарушений когнитивных функций у детей от матерей с СГТ, последние данные не выявили ассоциаций с повышением ТТГ во время беременности $[44,56]$. По данным датского когортного исследования, включившего 1153 женщины, ассоциации между повышением ТТГ в рамках 2,5-9,99 мЕд/л и результатами вербального IQ-теста, проведенного в возрасте 5 лет, получено не было [44]. В проспективном исследовании Nelsen S.M. et al. не выявили связи между СГТ (ТТГ 2,5-97,5-й процентиль) у женщин в I триместре беременности и способностью к обучению их детей в будущем [56].

\section{ЭФФЕКТИВНОСТЬ ЛЕЧЕНИЯ СУБКЛИНИЧЕСКОГО ГИПОТИРЕОЗА ВО ВРЕМЯ БЕРЕМЕННОСТИ И СОВРЕМЕННЫЕ ПОДХОДЫ К ТЕРАПИИ}

Оценить результаты исследований эффективности терапии левотироксином СГТ во время беременности зачастую представляется затруднительным из-за использования исследователями различных референсных значений ТТГ. В ретроспективном исследовании Maraka S. et al. принимали участие 5405 беременных с СГТ, который определялся как уровень ТТГ 2,5-10 мЕд/л. Из них 843 женщины получали лечение левотироксином. По данным исследователей, самопроизвольные аборты встречались на $38 \%$ реже в группе получающих лечение (ОШ 0,62; ДИ 95\% 0,48-0,82). Однако риск самопроизвольного аборта снижался на фоне лечения только женщин с уровнем ТТГ 4,1-10,0 мЕД/л (ОШ 0,45; ДИ 95\% 0,30-0,65), но не при ТТГ 2,5-4,0 (ОШ 0,91; ДИ 95\% 0,65-1,23) в сравнении с теми, кто не получал терапии. В то же время терапия тиреоидными гормонами ассоциировалась с большим риском преждевременных родов, ГСД и преэклампсии [57]. В иранском рандомизированном исследовании принимали участие 366 женщин с СГТ (ТТГ> 2,5 мЕд/л) и нормальным уровнем АТкТПО, половина из которых получали терапию левотироксином, и 1092 эутиреоидных женщин. Частота преждевременных родов не различалась между группами (ОР 86; 95\% ДИ 0,47-1,55; p=0,61), однако для женщин с ТТГ >4,0 мЕд/л было получено снижение частоты преждевременных родов в группе получавших лечение (ОР 0,38; 95\% ДИ 0,15-0,98; $p=0,04$ ) [58].

Другой проблемой оценки эффективности терапии СГТ во время беременности является позднее начало лечения в крупных рандомизированных исследованиях, что может вносить значимый вклад в отсутствие преимуществ для женщин, получающих лечение $[59,60]$. В рандомизированном исследовании Casey B.M. et al. принимали участие 677 беременных с СГТ (ТТГ $\geq 4,0$ мЕд/л и св.Т4 11-24 пмоль/л) или изолированной гипотироксинемией (ИГТ) (ТТГ 0,08-3,99 мЕд/л и св.Т4 <11 пмоль/л) на сроке до 20 нед гестации, которые были разделены на две группы. Одна группа получала терапию левотироксином с титрацией дозы до целевых значений ТТГ 0,1-2,5 мЕд/л, другая - плацебо. Статистических различий в отношении рисков неблагоприятных исходов беременности (самопроизвольные аборты, преждевременные роды, преэклампсия, ГСД, гестационная артериальная гипертензия), так же как и различий в когнитивных способностях детей в возрасте 3-5 лет, получено не было [59]. В рандомизированное исследование Lazarus J.H. et al. были включены 794 женщины на сроке до 16 нед беременности с СГТ (ТТГ >97,5 процентиля) или ИГТ (св.Т4 <2,5 процентиля). Из них 390 начали терапию левотироксином в дозе 150 мкг/сут, средний срок гестации при начале лечения составил 13 нед и 3 дня. Статистически значимых различий по уровню IQ в возрасте 3 лет у детей от матерей с тиреоидной патологией во время беременности получено не было [60]. К недостаткам данного исследования относятся позднее начало лечения, а также стартовая доза левотироксина, значительно превышающая общепринятую. B своей работе Zhao L. et al. исследова- 
ли вопрос показаний и сроков начала терапии левотироксином во время беременности. Участницы исследования (98 человек) были рандомизированы на три группы: 1-я группа начала терапию СГТ в I триместре, 2-я группа - во II триместре беременности, 3-я группа лечение не получала. СГТ в данном исследовании определялся как ТТГ > 2,5 мЕд/л в І триместре беременности и >3,0 мЕд/л во II триместре беременности при нормальном уровне св.Т4. Группы не различались по возрасту, ИМТ, уровню АТкТПО. В группе 1 реже наблюдались осложнения (преэклампсия, анемия, ГСД) $(9,7 \%)$ и неблагоприятные исходы (преждевременные роды, самопроизвольные аборты, замершие беременности, маловесные дети) беременности (3,2\%), чем группах 2 (41,9\% и 32,3\% соответственно) и 3 (64,5\% и 38,7\% соответственно), тогда как статистически значимые различия между исходами во 2-й и 3-й группах получены не были [61]. Есть мнение, что инициирование терапии левотироксином во II триместре беременности, когда щитовидная железа плода уже функционирует, не несет преимуществ $[43,61]$.

В настоящий момент в России отсутствуют единые стандарты ведения пациенток с патологией ЩЖ при планировании и в течение беременности. До недавнего времени СГТ во время беременности диагностировали при уровне ТТГ >2,5 мЕд/л в І триместре беременности, вне зависимости от наличия АТкТПО, согласно рекомендациям АTA 2011 и ЕTA 2014 гг. [27]. Более того, в клинической практике широкое распространение получило назначение левотироксина на прегравидарном этапе при уровне ТТГ >2,5 мЕд/л. Как мы видим из вышеизложенного обзора современной литературы, с точки зрения доказательной медицины такой подход приводит к гипердиагностике СГТ, избыточной лекарственной, психологической и финансовой нагрузке на беременных и систему здравоохранения.

В 2019 г. был опубликован проект российских клинических рекомендаций по лечению гипотиреоза, согласно которому не рекомендовано повсеместное лечение левотироксином женщин с ТТГ >2,5 мЕд/л во время беременности. В данном документе терапия левотироксином во время беременности рекомендована всем женщинам:

- с повышенным уровнем АТкТПО и ТТГ > 4 мЕд/л;

- с нормальным уровнем АТкТПО и ТТГ > 10 мЕд/л.

Лечение левотироксином может быть рассмотрено для женщин:

- с повышенным уровнем АТкТПО и ТТГ > 2,5 и <4 мЕд/л; - с нормальным уровнем АТкТПО и ТТГ >4,0 и <10 мЕд/л.

Мы можем видеть, что эффективность терапии левотироксином при СГТ (ТТГ 4,0-10 мЕД/л) в отсутствие аутоиммунного процесса в ЩЖ, так же как при высоконормальном уровне ТТГ (ТТГ 2,5-4,0 мЕД/л) у беременных с маркерами аутоиммунного тиреоидита, в настоящее время подвергнута сомнению $[2,13]$. В соответствии с проектом российских клинических рекомендаций решение вопроса о терапии левотироксином в таких случаях остается на усмотрение лечащего врача, поскольку убедительные данные за и против такого лечения отсутствуют [13]. Вероятно, даже после утверждения клинических рекомендаций по лечению гипотиреоза сохранится значительная разница подходов к терапии СГТ в связи с отсутствием достаточного количества научных данных для рекомендации однозначной стратегии в отношении СГТ во время беременности, а также в связи с инертностью в принятии новых клинических рекомендаций. Например, по данным Toloza F.J. et al., $52 \%$ опрошенных членов ATA продолжали использовать верхнюю границу референса ТТГ 2,5 мЕд/л для диагностики СГТ, несмотря на то, что 76\% опрошенных были ознакомлены с обновленными рекомендациями ATA 2017 г. [62].

Еще одной проблемой в настоящее время является отсутствие единого междисциплинарного подхода к диагностике и лечению СГТ между эндокринологами, акушерами-гинекологами, а во многих случаях и репродуктологами. Согласно клиническим рекомендациям Российского общества акушеров-гинекологов от 2019 г., консультация эндокринолога рекомендована при уровне ТТГ $\geq 3,0$ мЕд/л [63], что практическими врачами может рассматриваться как показания к терапии левотироксином.

\section{ЗАКЛЮЧЕНИЕ}

Современный взгляд на СГТ при планировании и во время беременности предполагает большую роль лечащего врача в выборе критериев диагностики и показаний к терапии. Тем важнее для клинического врача принимать осознанное и аргументированное решение о постановке диагноза и инициировании терапии, основываясь на доступных юридических документах и принципах доказательной медицины. Отсутствие единого подхода к диагностике и лечению СГТ у женщин репродуктивного возраста приводит к противоречивым назначениям, снижению доверия пациенток к лечащим врачам, увеличению психологического давления на беременную. Все вышеперечисленное диктует необходимость принятия единых критериев диагностики и лечения СГТ во время беременности.

\section{ДОПОЛНИТЕЛЬНАЯ ИНФОРМАЦИЯ}

Источник финансирования. Работа выполнена по инициативе авторов без привлечения финансирования.

Конфликт интересов. Авторы декларируют отсутствие явных и потенциальных конфликтов интересов, связанных с публикацией настоящей статьи.

Участие авторов. Шилова Е.С. - написание, редактирование и финальное утверждение рукописи; Боровик Н.В. - написание, редактирование и финальное утверждение рукописи; Попова П.В. - редактирование и финальное утверждение рукописи; Ярмолинская М.И. редактирование и финальное утверждение рукописи.

Все авторы одобрили финальную версию статьи перед публикацией, выразили согласие нести ответственность за все аспекты работы, подразумевающую надлежащее изучение и решение вопросов, связанных с точностью или добросовестностью любой части работы. 


\section{СПИСОК ЛИТЕРАТУРЫ | REFERENCES}

1. Lazarus J, Brown RS, Daumerie C et al. 2014 European thyroid association guidelines for the management of subclinical hypothyroidism in pregnancy and in children. Eur Thyroid J. 2014;3(2):76-94. doi: 10.1159/000362597

2. Alexander EK, Pearce EN, Brent GA, et al. 2017 Guidelines of the American Thyroid Association for the diagnosis and management of thyroid disease during pregnancy and the postpartum. Thyroid. 2017;27:315-89. doi: 10.1089/thy.2016.0457

3. Maraka S, Ospina NM, O'Keeffe DT et al. Subclinical Hypothyroidism in Pregnancy: A Systematic Review and Meta-Analysis. Thyroid. 2016;26(4):580-90. doi: 10.1089/thy.2015.0418

4. Korevaar TIM, Derakhshan A, Taylor PN, et al. Association of Thyroid Function Test Abnormalities and Thyroid Autoimmunity With Preterm Birth. JAMA. 2019;322(7):632. doi: https://doi.org/10.1001/jama.2019.10931

5. Chen L, Hu R. Thyroid autoimmunity and miscarriage: a meta-analysis. Clinical Endocrinology. 2011;74(4):513-519. doi: 10.1111/j.1365-2265.2010.03974.x

6. Liu H, Shan Z, Li,C, et al. Maternal Subclinical Hypothyroidism, Thyroid Autoimmunity, and the Risk of Miscarriage: A Prospective Cohort Study. Thyroid. 2014;24(11):1642-1649. doi: 10.1089/thy.2014.0029

7. Seungdamrong A, Steiner AZ, Gracia CR, et al. Eunice Kennedy Shriver National Institute of child health and human development reproductive medicine network. Preconceptional antithyroid peroxidase antibodies, but not thyroid-stimulating hormone, are associated with decreased live birth rates in infertile women. Fertil Steril. 2017;108:843-50. doi: 10.1016/j.fertnstert.2017.08.026

8. Thangaratinam S, Tan A, Knox E, et al. Association between thyroid autoantibodies and miscarriage and preterm birth: metaanalysis of evidence. BMJ. 2011;342:d2616. doi: 10.1136/bmj.d2616.

9. Van den Boogaard E, Vissenberg R, Land JA, et al. Significance of (sub) clinical thyroid dysfunction and thyroid autoimmunity before conception and in early pregnancy: a systematic review. Human Reproduction Update. 2011;17(5):605-619. doi: 10.1093/humupd/dmr024

10. Negro R. Thyroid autoimmunity and pre-term delivery: brief review and meta-analysis. J Endocrinol Invest. 2011; 34:155-158. doi: 10.1007/BF03347047

11. He X, Wang P, Wang Z, He X, et al. Thyroid antibodies and risk of preterm delivery: a metaanalysis of prospective cohort studies. Eur J Endocrinol. 2012;167:455-464. doi: 10.1530/EJE-12-0379.

12. Korevaar TM, Pop VJ, Chaker L, et al. Dose dependency and a functional cutoff for TPO-antibody positivity during pregnancy. J Clin Endocrinol Metab. 2018;103:778-789. doi: 10.1210/jc.2017-01560

13. Дедов И.И., Мельниченко Г.А. и др. Клинические рекомендации Российской ассочиачии эндокринологов. Гипотиреоз (проект). 2019. [Dedov II, Melnichenko GA, et al. Clinical Guidelines of Russian Association of Endocrinologists. Hypothyroidism. 2019. (In Russ.)]. Доступно по: https://www.endocrincentr.ru/sites/default/files/ specialists/science/clinic-recomendations/gipotireoz.proekt.klin_. rek_3.pdf Ссылка активна на 05.10.20 г.

14. Soldin OP, Tractenberg RE, Hollowell JG, et al. Trimester-Specific Changes in Maternal Thyroid Hormone, Thyrotropin, and Thyroglobulin Concentrations During Gestation: Trends and Associations Across Trimesters in lodine Sufficiency. Thyroid. 2004;14(12):1084-1090. doi: 10.1089/thy.2004.14.1084

15. Haddow JE, McClain M, Lambert-Messerlian G, et al. Variability in thyroid stimulating hormone suppression by human chronic gonadotropin during early pregnancy. J Clin Endocrinol Metab. 2008;93:3341- 3347. doi: 10.1210/jc.2008-0568

16. Garber JR, Cobin RH, Gharib H, et al. American Association of Clinical Endocrinologists and American Thyroid Association Taskforce on Hypothyroidism in Adults. Clinical practice guidelines for hypothyroidism in adults: cosponsored by the American Association of Clinical Endocrinologists and the American Thyroid Association. Endocr Pract. 2012;18(6):988-1028. doi: 10.4158/EP12280.GL

17. Elenkov IJ, Wilder RL, Bakalov VK,et al. IL-12, TNF-a, and hormonal changes during late pregnancy and early postpartum: implications for autoimmune disease activity during these times. J Clin Endocrinol Metab. 2001;86(10):4933-4938. doi: 10.1210/jcem.86.10.7905

18. Zimmermann MB. The effects of iodine deficiency in pregnancy and infancy. Paediatr. Perinat Epidemiol. 2012;26(1):108-117. doi: $10.1111 / j .1365-3016.2012 .01275 . x$.
19. Соболева Д.Е., Дора С.В., Волкова А.Р. и др. Йодообеспечение беременных женщин Санкт-Петербурга. Эффективность профилактики развития йододефицитных заболеваний // Проблемы женского здоровья. — 2015. - № 2. - Т. 10. C. 25-31. [Soboleva DE, Dora SV, Volkova AR, et al. lodine status of pregnant women living in Saint Petersburg. The effectiveness of iodine prevention in risk group. Problems of Women Health. 2015;10(2): 25-31. (In Russ.)].

20. Benhadi $\mathrm{N}$, et al. Ethnic differences in TSH but not in free T4 concentrations or TPO antibodies during pregnancy. Clin. Endocrinol. (Oxf.). 2007;66:765-770. doi: 10.1111/j.1365-2265.2007.02803.x

21. Korevaar TI, Steegers EA, de Rijke YB. et al. Hypothyroxinemia and TPO-antibody positivity are risk factors for premature delivery: the generation R study. Clin. Endocrinol. Metab. 2013;98:4382-4390. doi: 10.1210/jc.2013-2855

22. Han C, et al. High body mass index is an indicator of maternal hypothyroidism, hypothyroxinemia, and thyroid-peroxidase antibody positivity during early pregnancy. Biomed. Res. Int. 2015;321831. doi: $10.1155 / 2015 / 351831$

23. Mosso $\mathrm{L}$, et al. Early pregnancy thyroid hormone reference ranges in Chilean women: the influence of body mass index. Clin. Endocrinol. (Oxf.). 2016:85:942-948. doi: 10.1111/cen.13127

24. Korevaar TI, Steegers EAP, Pop VJ, et al. Thyroid autoimmunity impairs the thyroidal response to human chorionic gonadotropin: two population-based prospective cohort studies. J. Clin. Endocrinol. Metab. 2017:102:69-77. doi: 10.1210/jc.2016-2942

25. Hou Y, Liu A, Li J, et al. Different Thyroidal Responses to Human Chorionic Gonadotropin Under Different Thyroid Peroxidase Antibody and/or Thyroglobulin Antibody Positivity Conditions During the First Half of Pregnancy. Thyroid. 2019 Apr; 29(4):577-585. doi: 10.1089/thy.2018.0097

26. Korevaar Tl, Steegers EA, de Rijke YB, et al. Placental angiogenic factors are associated with maternal thyroid function and modify hCG-mediated FT4 stimulation. J. Clin. Endocrinol. Metab. 2015;100:E1328-E2334. doi: 10.1210/jc.2015-2553

27. Stagnaro-Green A, Abalovich M, Alexander E, et al. Guidelines of the American Thyroid Association for the Diagnosis and Management of Thyroid Disease During Pregnancy and Postpartum. Thyroid. 2011;21(10):1081-1125. doi:10.1089/thy.2011.0087

28. Canovi S, Vezzani S, Polese A, et al. Pregnancy-related reference intervals for serum thyrotropin based on real-life clinical data. Gynecol Endocrinol. 2020;322(7):1-4. doi: https://doi.org/10.1080/09513590.2020.1756251

29. Blatt AJ, Nakamoto JM, Kaufman HW. National status of testing for hypothyroidism during pregnancy and postpartum. J Clin Endocrinol Metab. 2012;97:777-784. doi: 10.1210/jc.2011-2038

30. Li C, Shan Z, Mao J, et al. Assessment of Thyroid Function During First-Trimester Pregnancy: What Is the Rational Upper Limit of Serum TSH During the First Trimester in Chinese Pregnant Women? The Journal of Clinical Endocrinology \& Metabolism. 2014;9(1):73-79. doi: 10.1210/jc.2013-1674

31. Medici $\mathrm{M}$, Korevaar TI, Visser WE, et al. Thyroid function in pregnancy: what is normal? Clin Chem. 2015;61(5):704-713. doi: 10.1373/clinchem.2014.236646

32. Попова П.В., Шилова Е.С., Ткачук А.С., и др. Референтные значения показателей функции щитовидной железы в первом триместре беременности и риск развития гестационного сахарного диабета у женщин Санкт-Петербурга // Сахарный диабет. - 2018. - Т. 21. №1. - C. 34-41. [Popova PV, Shilova ES, Tkachuk AS. First Trimester Thyroid Function in Pregnant Women residing in St Petersburg (Russia): Reference Values and Risk of Gestational Diabetes. Diabetes Mellitus. 2018;21(1):34-41. (In Russ.)]. doi: 10.14341/DM9283

33. Krassas GE, Pontikides N, Kaltsas T, et al. Disturbances of menstruation in hypothyroidism. Clin Endocrinol (Oxf). 1999;50(5):655-659. doi: 10.1046/j.1365-2265.1999.00719.x

34. Arojoki M, Jokimaa V, Juuti A, et al. Hypothyroidism among infertile women in Finland. Gynecol Endocrinol. 2000;14(2):127-131. doi: 10.3109/09513590009167671

35. Abalovich M, Mitelberg L, Allami C, et al. Subclinical hypothyroidism and thyroid autoimmunity in women with infertility. Gynecol Endocrinol. 2007;23(5):279-283. doi: 10.1080/09513590701259542

36. Feldthusen $\mathrm{AD}$, Pedersen PL, Larsen J, et al. Impaired fertility associated with subclinical hypothyroidism and thyroid autoimmunity: the Danish General Suburban Population Study. J Pregnancy. 2015;2015:132718. doi: 10.1155/2015/132718 
37. Poppe K, Glinoer D, Van Steirteghem A, et al. Thyroid dysfunction and autoimmunity in infertile women. Thyroid. 2002;12(11):997-1001. doi: 10.1089/105072502320908330

38. Plowden TC, Schisterman EF, Sjaarda LA, et al. Subclinical hypothyroidism and thyroid autoimmunity are not associated with fecundity, pregnancy loss, or live birth. J Clin Endocrinol Metab. 2016:101(6):2358-2365. doi: 10.1210/jc.2016-1049

39. Practice Committee of the American Society for Reproductive Medicine. Subclinical hypothyroidism in the infertile female population: a guideline. Fertil Steril. 2015;104(3):545-553. doi: 10.1016/j.fertnstert.2015.05.028

40. Dhillon-Smith RK, Middleton LJ, Sunner KK, et al. Levothyroxine in Women with Thyroid Peroxidase Antibodies before Conception. N Engl J Med. 2019;380(14):1316-1325. doi: 10.1056/NEJMoa1812537.

41. Kim CH, Ahn JW, Kang SP, et al. Effect of levothyroxine treatment on in vitro fertilization and pregnancy outcome in infertile women with subclinical hypothyroidism undergoing in vitro fertilization/ intracytoplasmic sperm injection. Fertil Steril. 2011;95(5):1650-1654. doi: 10.1016/.jertnstert.2010.12.004

42. Wang $\mathrm{H}, \mathrm{Gao} \mathrm{H}$, Chi H, et al. Effect of levothyroxine on miscarriage among women with normal thyroid function and thyroid autoimmunity undergoing in vitro fertilization and embryo transfer: a randomized clinical trial. JAMA. 2017:318(22):2190-2198. doi: 10.1001/jama.2017.18249.

43. Dong AC, Stephenson MD, Stagnaro-Green AS. The Need for Dynamic Clinical Guidelines: A Systematic Review of New Research Published After Release of the 2017 ATA Guidelines on Thyroid Disease During Pregnancy and the Postpartum. Frontiers in Endocrinology. 2020;1 1. doi: 10.3389/fendo.2020.00193

44. Andersen SL, Andersen S, Liew Z, Vestergaard P, Olsen J. Maternal thyroid function in early pregnancy and neuropsychological performance of the child at 5 years of age. J Clin Endocrinol Metab. 2018;103:660-70. doi: 10.1210/jc.2017-02171

45. Andersen SL, Andersen S, Vestergaard P, Olsen J. Maternal thyroid a Danish Nationwide Case-Cohort Study. Thyroid. 2018;28:537-46. doi: 10.1089/thy.2017.0425

46. Yang J, Liu Y, Liu H, et al. Associations of maternal iodine status and thyroid function with adverse pregnancy outcomes in Henan Province of China. J Trace Elem Med Biol. 2018;47:104-10. doi: 10.1016/j.jtemb.2018.01.013

47. Negro R, Schwartz A, Gismondi R, et al. Increased Pregnancy Loss Rate in Thyroid Antibody Negative Women with TSH Levels between 2.5 and 5.0 in the First Trimester of Pregnancy. The Journal of Clinical Endocrinology \& Metabolism. 2010;95(9):E44-E48. doi:10.1210/jc.2010-0340

48. Schneuer FJ, Nassar N, Tasevski V, et al. Association and predictive accuracy of high TSH serum levels in first trimester and adverse pregnancy outcomes. J Clin Endocrinol Metab. 2012;97(9):3115-3122. doi: 10.1210/jc.2012-1193

49. Wang S, Teng WP, Li JX, et al. Effects of maternal subclinical hypothyroidism on obstetrical outcomes during early pregnancy. J Endocrinol Invest. 2012;35(3):322-325. doi: 10.3275/7772

50. Lopez-Tinoco C, Rodriguez-Mengual A, Lara-Barea A, et al. Impact of positive thyroid autoimmunity on pregnant women with subclinical hypothyroidism. Endocrinol Diabetes Nutr. 2018;65:150-5. doi: 10.1016/j.endien.2017.11.020
51. Arbib N, Hadar E, Sneh-Arbib O, et al. First trimester thyroid stimulating hormone as an independent risk factor for adverse pregnancy outcome. J Matern Fetal Neonatal Med. 2017:30:2174-8. doi: 10.1080/14767058.2016.1242123

52. Derakhshan A, Peeters RP, Taylor PN, et al. Association of maternal thyroid function with birthweight: a systematic review and individualparticipant data meta-analysis. The Lancet Diabetes \& Endocrinology. 2020;8(6):501-510. doi: 10.1016/s2213-8587(20)30061-9

53. Chen LM, Du WJ, Dai J, et al. Effects of subclinical hypothyroidism on maternal and perinatal outcomes during pregnancy: a single-center cohort study of a Chinese population. PLoS One. 2014;9(10):e109364. doi: 10.1371/journal.pone.0109364

54. Wilson KL, Casey BM, McIntire DD, et al. Subclinical thyroid disease and the incidence of hypertension in pregnancy. Obstet Gynecol. 2012;119(2Pt1):315-320. doi: 10.1097/AOG.0b013e318240de6a

55. Tudela CM, Casey BM, McIntire DD, et al. Relationship of subclinical thyroid disease to the incidence of gestational diabetes. Obstet Gynecol. 2012:119(5):983-988. doi: 10.1097/AOG.0b013e318250aeeb

56. Nelson SM, Haig C, McConnachie A, et al. Maternal thyroid function and child educational attainment: prospective cohort study. BMJ. 2018;360:k452. doi: 10.1136/bmj.k452

57. Maraka S, Mwangi R, McCoy RG, et al. Thyroid hormone treatment among pregnant women with subclinical hypothyroidism: US national assessment. BMJ. 2017;356:i6865. doi: 10.1136/bmj.i6865

58. Nazarpour S, Ramezani Tehrani F, Simbar M, et al. Effects of levothyroxine on pregnant women with subclinical hypothyroidism, negative for thyroid peroxidase antibodies. J Clin Endocrine Metab. 2018;103:926-35. doi: 10.1210/jc.2017-0185

59. Casey BM, Thom EA, Peaceman AM, et al. Treatment of Subclinical Hypothyroidism or Hypothyroxinemia in Pregnancy. New England Journal of Medicine. 2017;376(9):815-825. doi: 10.1056/nejmoa1606205

60. Lazarus JH, Bestwick JP, Channon S et al. Antenatal thyroid screening and childhood cognitive function. N Engl J Med. 2012;366(6):493-501. doi: 10.1056/NEJMoa1 106104

61. Zhao L, Jiang G, Tian $X$, et al. Initiation timing effect of levothyroxine treatment on subclinical hypothyroidism in pregnancy. Gynecol Endocrinol. 2018;34:845-8. doi: 10.1080/09513590.2018.1451836

62. Toloza FJK, Singh Ospina NM, Rodriguez-Gutierrez R, et al. Practice Variation in the Care of Subclinical Hypothyroidism During Pregnancy: A National Survey of Physicians in the United States. J Endocr Soc. 2019;3(10):1892-1906. doi: https://doi.org/10.1210/js.2019-00196

63. Долгушина Н.В., Артымук Н.В., Белокриницкая Т.Е. и др. Клинические рекомендации Российского общества акушеровгинекологов. Нормальная беременность. 2019. [Dolgushina NV, Artimuk NV, Belokrinitskaya TE, et al. Clinical Guidelines of Russian Association of Obstetrician and Gynecologist. Physiological Pregnancy. 2019. (In Russ.)]. Доступно по: https://docviewer.yandex ru/view/0/? ${ }^{*}=$ KKnu28OZjp5ygElEAiA07g8NBZt7InVybCI6InlhLWRpc 2stcHVibGljOi8VVzZJSFh1VWpLdERmSIRIb11xSjk2UHQVdGRIL1 ovL3 VXM1dCUFV1WCsrMXVCTkJBVC9mVW9OSjA4VXZaYVJTMnEvSjZicG 1SeU9Kb25UM1ZvWG5EYWc9PSIsInRpdGXIljoiOJ3QvtGAOLzQsNC7 OYzQvdCw0Y8g0LHQtdGAOLXQvNC10L3QvdC\%2BOYHRgtGMLmR vY3giLCJub2ImcmFtZSI6ZmFsc2UsInVpZCI6ljAiLCJ0cyl6MTYwMTg5 NDU1MDkwOCwieXUiOil1 Mzk2NjAyOTMxNTk5MDMzNjQzIn0\%3D Ссылка активна на 05.10.20 г.

Рукопись получена: 07.10.2020. Одобрена к публикации: 03.12.2020. Опубликована online: 30.12.2020.

ИНФОРМАЦИЯ ОБ АВТОРАХ [AUTHORS INFO]

*Шилова Екатерина Сергеевна [Ekaterina S. Shilova]; адрес: 199034, Санкт-Петербург, Менделеевская линия, д. 3 [address: 199034, St. Petersburg, Mendeleyevskaya Line, 3]; ORCID: https://orcid.org/0000-0002-5225-6054; eLibrary SPIN: 9703-5970; e-mail: katia.shilova@gmail.com.

Боровик Наталья Викторовна, к.м.н. [Natalya V. Borovik, MD, PhD]; e-mail: borovik1970@yandex.ru; ORCID: https://orcid.org/0000-0003-0835-6741; eLibrary SPIN: 9010-7276.

Попова Полина Викторовна, к.м.н. [Polina V. Popova, MD, PhD]; e-mail: pvpopova@yandex.ru; ORCID: https://orcid.org/0000-0002-3697-7791; eLibrary SPIN: 1150-3432.

Ярмолинская Мария Игоревна, д.М.н., професcop [Maria I. Yarmolinskaya, MD, PhD, Professor]; e-mail: m.yarmolinskaya@gmail.com; ORCID: https://orcid.org/0000-0002-6551-4147; eLibrary SPIN: $3686-3605$. 


\section{ЦИТИРОВАТЬ:}

Шилова Е.С., Боровик Н.В., Попова П.В., Ярмолинская М.И. Диагностика и лечение субклинического гипотиреоза при планировании и во время беременности: современный подход к проблеме // Проблемы эндокринологии. - 2020. T. 66. — №6. — C. 65-73. doi: https://doi.org/10.14341/probl12687

\section{TO CITE THIS ARTICLE:}

Shilova ES, Borovik NV, Popova PV, Yarmolinskaya MI. Diagnostic and treatment of subclinical hypothyroidism in pregnant and planning pregnancy patients: modern view of the problem. Problems of Endocrinology. 2020;66(6):65-73. doi: https://doi.org/10.14341/probl12687 